\title{
Neoadjuvant chemotherapy with radical surgery vs radical surgery alone for cervical cancer: a systematic review and meta-analysis
}

This article was published in the following Dove Medical Press journal: OncoTargets and Therapy

\section{Hui Zhao \\ Yue $\mathrm{He}$ \\ Shu-Li Yang \\ Qun Zhao \\ Yu-Mei Wu}

Department of Gynecological Oncology, Beijing Obstetrics and Gynecology Hospital, Capital Medical University, Beijing, China
Correspondence: Yu-Mei Wu Department of Gynecological Oncology, Beijing Obstetrics and Gynecology Hospital, Capital Medical University, No 17 Qihelou Street, Dongcheng District, Beijing 100006, China Tel +86 I05 2277296 Emailwym597118@163.com
Aim: This systematic review was designed to evaluate the efficacy of neoadjuvant chemotherapy with radical surgery vs radical surgery alone for cervical cancer.

Methods: A computerized search was done for trials from PubMed, EMBASE, CENTRAL, and Cochrane Database of Systematic Reviews. The trials included neoadjuvant chemotherapy plus radical surgery vs radical surgery alone. We measured overall survival (OS), disease-free survival (DFS), progression-free survival (PFS), local and distant recurrence, lymph node metastasis, and parametrial infiltration per patient.

Results: In all, 13 studies involving 2,158 subjects were included. In regard to OS, DFS, PFS, local and distant recurrence, and parametrial infiltration, neoadjuvant chemotherapy plus radical surgery was similar to radical surgery alone. Among them, subgroup analysis of eight studies involving 1,544 patients with locally advanced cervical cancer (FIGO stage IB2-IIB) showed that neoadjuvant chemotherapy (NACT) plus radical surgery significantly improved OS, and decreased local and distant recurrence rates, lymph node metastasis rate, and the level of parametrial infiltration compared to radical surgery alone.

Conclusion: The present study demonstrates that preoperative NACT is now an accepted effective procedure in selected patients with locally advanced cervical cancer (FIGO stage IB2-IIB). However, the relationship between NACT and longer DFS and PFS cannot be demonstrated by this meta-analysis. Thus, the decision to use or not to use NACT before radical surgery depends on the surgeon's experience and clinical judgment. Nevertheless, further research in this field is urgently needed to confirm it.

Keywords: neoadjuvant chemotherapy, surgery, cervical cancer, meta-analysis

\section{Introduction}

Cervical cancer is one of the common malignant tumors that affect the health of females all over the world. Its morbidity and mortality are ranking second in oncology in the world and ranking fourth in oncology for women. ${ }^{1}$ For early stage cervical cancer, surgery and radiotherapy are the standard treatment modalities. ${ }^{2}$ In order to further improve the prognosis, comprehensive treatment regimens, mainly including surgery, radiotherapy, and chemotherapy, have been developed, with continuous exploration by investigators over recent years. ${ }^{3,4}$ However, the best treatment is still controversial.

Previous observations show that cervical cancer is moderately chemosensitive. Investigators generally believe that only when other treatment options fail, will advanced or recurrent patients choose chemotherapy as a treatment. In recent years, the age of onset of cervical cancer has tended to be younger and the proportion of adenocarcinoma has increased, but young patients have higher ovarian retention 
and sexual function requirements. While traditional simple radical radiotherapy and reoperation after radiotherapy for squamous cell carcinoma are good, there was no significant increase in the survival rate of cervical adenocarcinoma patients, especially for bulky (tumor diameter $\geq 4 \mathrm{~cm}$ ) and locally advanced cervical cancer patients. Since the late 1980s, preoperative neoadjuvant chemotherapy (NACT) has been proposed and carried out. ${ }^{5}$ NACT as one of the adjuvant treatments of cervical cancer reduces tumor volume, kills subclinical lesions, increases tumor resectability, eliminates micro-metastases, and has received widespread attention. There have been numerous published studies on neoadjuvant chemotherapy for cervical cancer, but the results differ. Therefore, it is necessary to conduct a more rigorous, comprehensive, and objective systematic review of the efficacy of neoadjuvant chemotherapy. Therefore, the aim of this study was to compare the long-term efficacy of neoadjuvant chemotherapy followed by surgery vs surgery alone for patients with early-stage or locally advanced cervical cancer (FIGO stage IB2, stage IIA, stage IIB), and provide a theoretical basis for the clinical application of NACT.

\section{Patients and methods}

This meta-analysis was performed according to the Cochrane Handbook for Systematic Reviews of Interventions ${ }^{6}$ and presented based on Preferred Reporting Items for Systematic Reviews and Meta-Analyses guidelines. ${ }^{7}$

\section{Search strategy}

We conducted a systematic search of PubMed, EMBASE, Cochrane Central Register of Clinical Trials (CENTRAL), and Cochrane Database of Systematic Reviews, using the $\mathrm{MeSH}$ terms and free key words "cervical cancer", "surgery", and "chemotherapy", from their dates of inception to October 2017, and identified all potentially relevant articles; there was no language restriction. We also searched the reference lists of the full-text papers and reviewed studies from all of the relevant publications to identify any omitted studies.

\section{Inclusion criteria}

Inclusion criteria were articles related to: 1) patients who were diagnosed with early or locally advanced cervical cancer and were treatment naive; 2) trials focused on comparing neoadjuvant chemotherapy plus radical surgery with radical surgery alone; 3 ) at least one of the outcome measures mentioned below was reported: overall survival (OS), diseasefree survival (DFS), progression-free survival (PFS), local and distant recurrence, lymph node metastasis, or parametrial infiltration; and 4) randomized controlled trials (RCTs), prospective non-RCTs, or retrospective clinical control study. Articles with the following exclusion criteria were eliminated: 1) trials without a placebo or treatment group; 2 ) the reported data was clearly erroneous or incomplete, and was unable to provide research outcomes; 3 ) case reports, or observational studies; and 4) duplicated publications.

\section{Risk-of-bias assessments}

The risk of bias in each included study was evaluated based on Cochrane handbook version 5.1.0 for Systematic Reviews by the Cochrane Collaboration. Study quality was evaluated, including random sequence generation, allocation concealment, blinding of participants and personnel, blinding of outcome assessment, incomplete outcome data, selective reporting, and other biases. Each entry was then classified as "low risk", "unclear risk", or "high risk".

\section{Data selection and extraction}

Trials identified through the search activities described above were each assigned to a review topic (or topics). Data extracted from the review were entered into Thomson Research Software (EndNote X4), and checked for accuracy. When information regarding any of the above was unclear, original reports would be consulted for further details. "Included", "pending", "excluded (reason)" were indicated in the "notes" column, and "pending" reports were retraced from the references.

A self-designed data extraction form was used by two researchers to independently extract content, including lead author, year of publication, participant characteristics, the clinical stage of cancer, histological type, tumor size, treatment measures, outcomes, effect indicators, and follow-up duration. Literature screening, quality evaluation, and data extraction were carried out by two reviewers. In cases of disagreement, a third investigator helped to resolve the disagreement through discussion.

\section{Statistical analysis}

Review Manager Software (RevMan5.3, offered by the Cochrane Collaboration) was used for statistical analysis. Odds ratios (ORs) and 95\% CIs were used for binary data meta-analysis of effect size. The chi-squared test was used to assess significance of heterogeneity, and the degree of heterogeneity was then examined using the $I^{2}$ statistic. The fixed-effects model was used if the assessment of heterogeneity was not significant $\left(P>0.1, I^{2} \leq 50 \%\right)$. If the source of 
heterogeneity was uncertain, the random-effects model was used for analysis.

\section{Results}

\section{Study selection}

A total of 501 articles were retrieved. Thirty-five duplicate publications were excluded and 446 irrelevant papers were excluded based on a review of titles and abstracts. An intensive reading full-text review of the 20 included articles further removed seven articles. Finally, a total of 13 studies, ${ }^{8-20}$ published between 1997 and 2016, were assessed for eligibility in the meta-analysis (Figure 1).

\section{Quality assessment}

Three studies ${ }^{10,17,19}$ used random number tables and computergenerated random sequence for random sequence generation, while four studies ${ }^{10-12,18}$ just reported the randomized trials and provided no description of randomization methods. Six studies of random grouping methods were assessed as a high risk of bias. Two trials ${ }^{10,19}$ allocated patient concealment by sealed envelopes. Blinded methods were neither used in participants nor intervention providers because of the nature of the interventions. Most trials had comparable baselines, except one trial ${ }^{9}$ with a statistically significant difference between the two groups for FIGO stage. Blinding of outcome assessment independent of the trial was done in six studies. ${ }^{10-13,17,19}$ None of the included studies had an incomplete report or a selective report. Overall, three studies ${ }^{10,17,19}$ were of high methodological quality, four ${ }^{10-12,18}$ were of moderate quality, and the remaining were of low quality. Summaries of the quality assessment results for all of the trials are shown in Figures 2 and 3.

\section{Characteristics of study selection}

Totally, 2,158 cervical cancer patients were included in this meta-analysis, 814 receiving NACT plus radical surgery, and 1,344 receiving radical surgery alone. Studies included women with FIGO stage IB-IIB cervical cancer. Among those patients, the mean age ranged from 20-77 years, the sample size ranged from 60-524, the follow-up period varied from 37-94 months, and there was no statistically significant difference between the two groups for tumor's histological type. Intervention strategies were similar among most of the studies. The characteristics of included studies are depicted in Table 1.

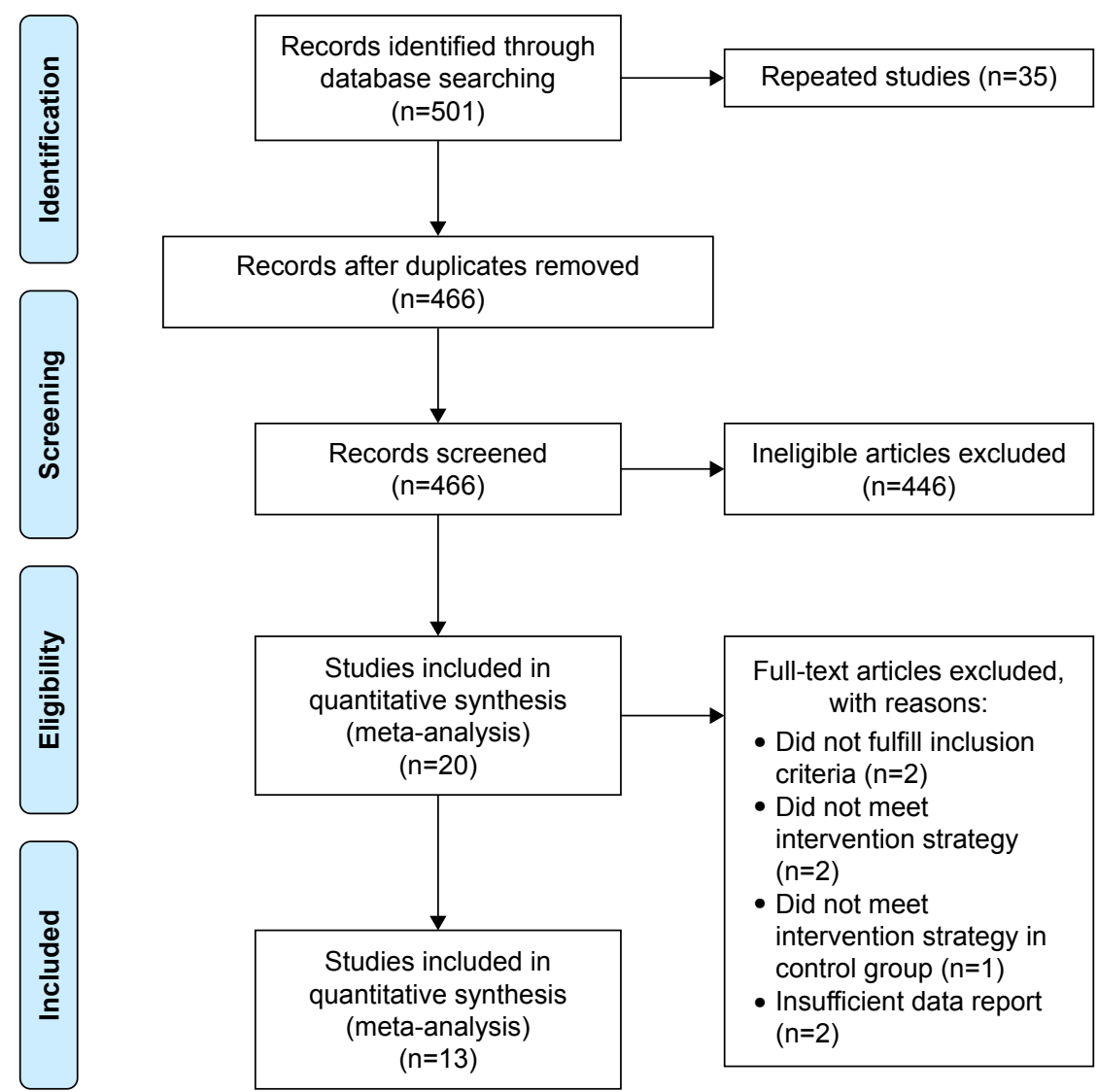

Figure I Flow diagram of study searching strategy. 


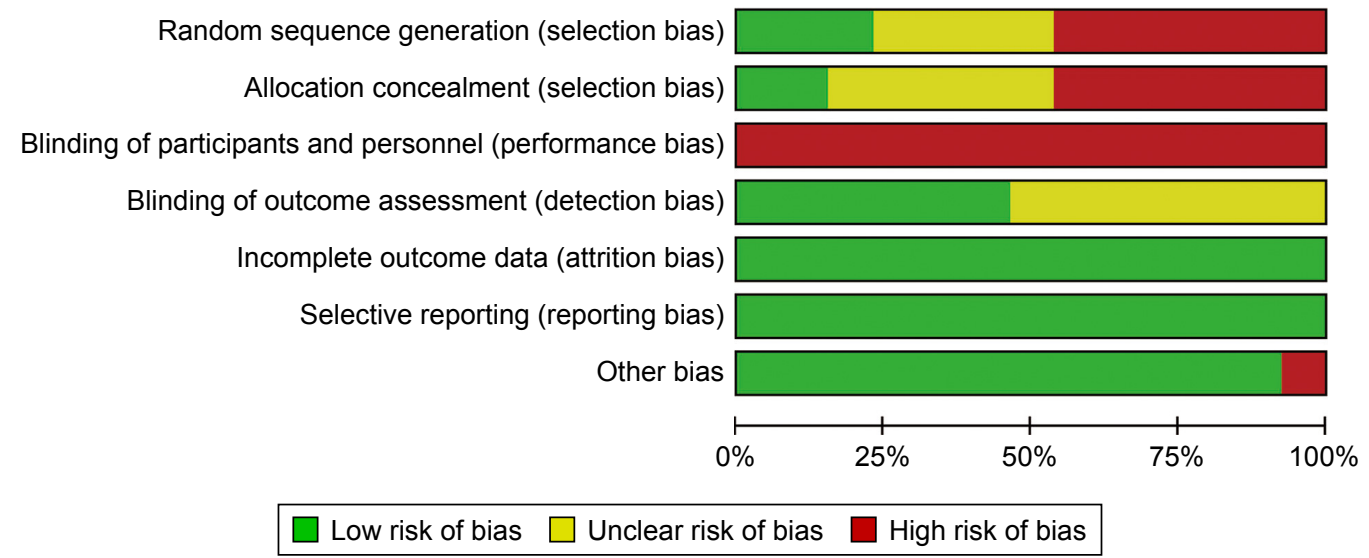

Figure 2 Quality assessment summary for included studies.

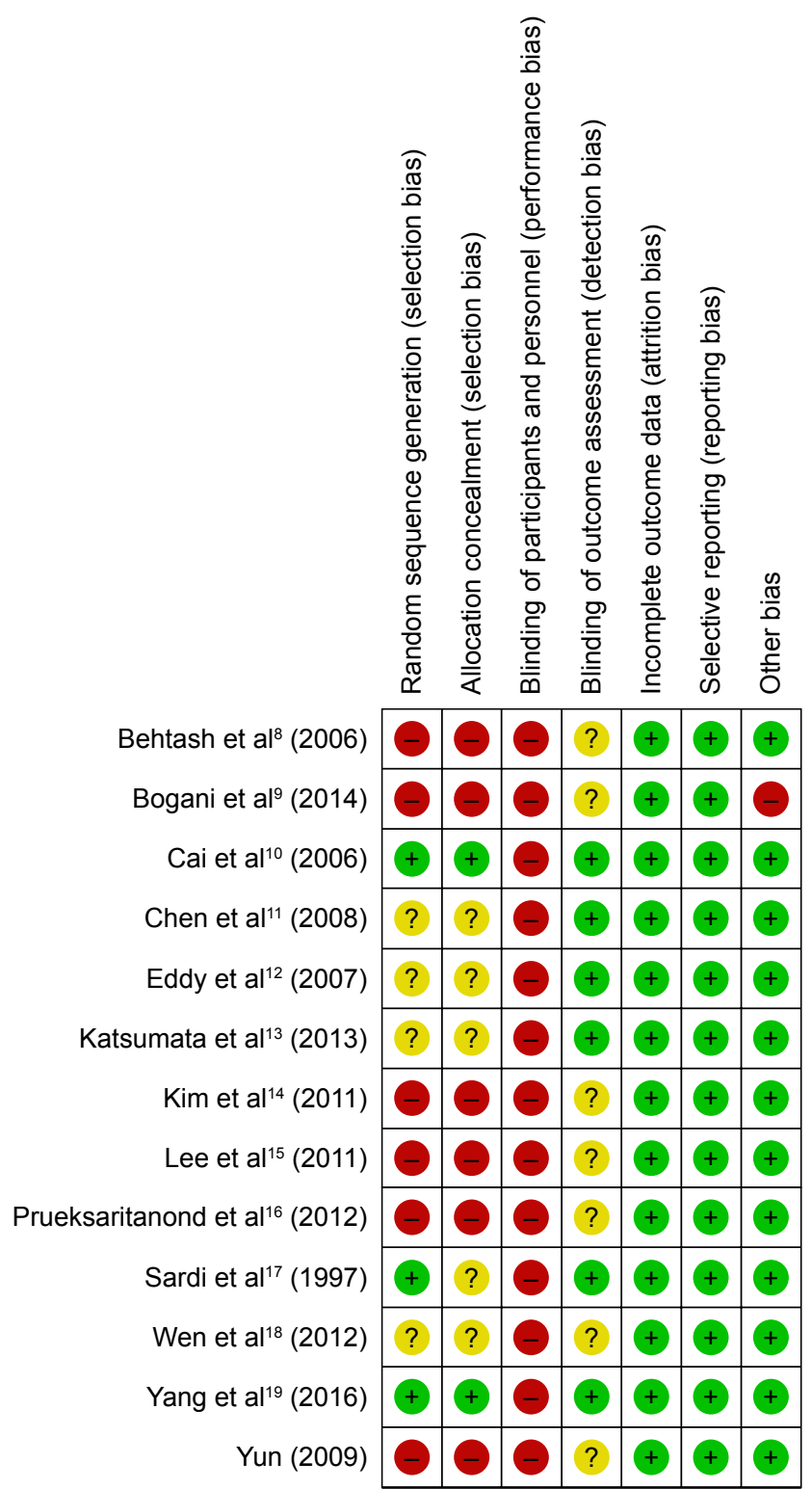

Figure 3 Methodological quality assessment for each included study. Note: +, low risk of bias; -, high risk of bias; ?, unclear risk of bias.

\section{Outcomes and synthesis of results Effects of interventions for early cervical cancer}

\section{Overall survival}

Eleven studies ${ }^{8-13,15,17-20}$ reported OS, including a total of 1,554 patients (701 in the NACT+radical surgery group and 853 in the radical surgery alone group). There was no statistical between-study heterogeneity in OR of studies ( $P=0.08, I^{2}=40 \%$ ); we used the fixed effects model for merging. The pooled estimates of effect sizes showed no statistical difference in OS between the two groups $(\mathrm{OR}=1.21,95 \% \mathrm{CI}=0.94-1.55, P=0.14)$, as shown in Figure 4.

\section{Disease-free survival}

Six studies ${ }^{8,10-12,19,20}$ reported DFS, including a total of 1,021 patients (449 in the NACT+radical surgery group and 572 in the radical surgery alone group). There was statistical between-study heterogeneity in OR of studies $(P=0.003$, $I^{2}=72 \%$ ); we used the random effects model for merging. The pooled estimates of effect sizes showed no statistical difference in DFS between the two groups $(\mathrm{OR}=1.15,95 \%$ $\mathrm{CI}=0.60-2.21, P=0.67$ ), as shown in Figure 4.

\section{Progression-free survival}

Four studies ${ }^{12,13,15,17}$ reported PFS, including a total of 556 patients (275 in the NACT+radical surgery group, and 281 in the radical surgery alone group). There was no statistical between-study heterogeneity in the OR of studies ( $P=0.45, I^{2}=0 \%$ ); we used the fixed effects model for merging. The pooled estimates of effect sizes showed no statistical difference in PFS between the two groups $(\mathrm{OR}=1.10$, $95 \% \mathrm{CI}=0.77-1.57, P=0.60)$, as shown in Figure 4. 


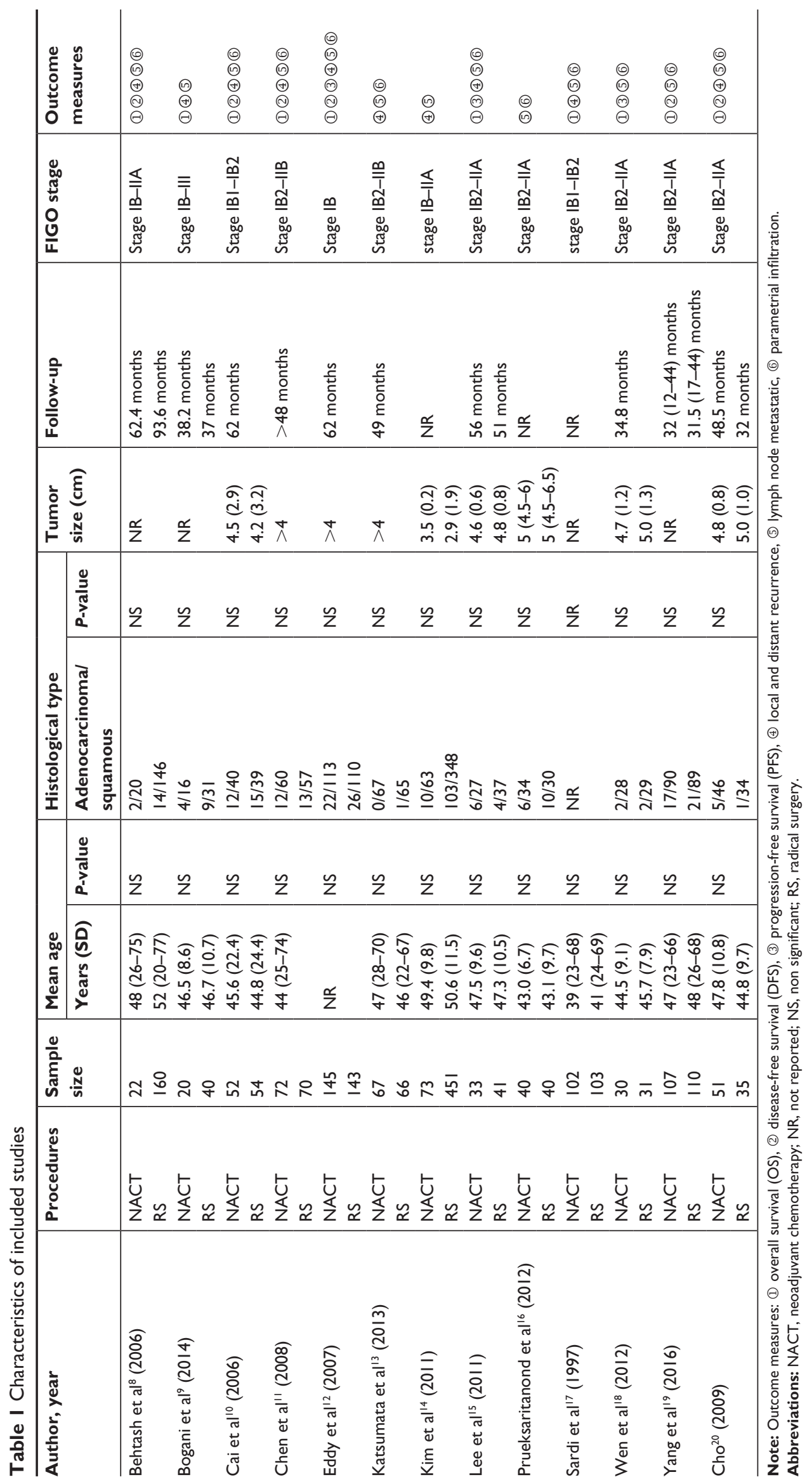




\section{A}

\begin{tabular}{|c|}
\hline Study or subgroup \\
\hline Behtash et al ${ }^{8}(2006)$ \\
\hline Bogani et al ${ }^{9}(2014)$ \\
\hline Cai et al ${ }^{10}(2006)$ \\
\hline Chen et al ${ }^{11}(2008)$ \\
\hline Eddy et al ${ }^{12}(2007)$ \\
\hline Katsumata et al ${ }^{13}(201$ \\
\hline Lee et $\mathrm{al}^{15}(2011)$ \\
\hline Sardi et al ${ }^{17}$ (1997) \\
\hline Wen et $a^{18}(2012)$ \\
\hline Yang et al ${ }^{19}(2016)$ \\
\hline $\mathrm{Cho}^{20}(2009)$ \\
\hline
\end{tabular}

Events

Weight Odds ratio

$\mathrm{M}-\mathrm{H}$, fixed, $95 \% \mathrm{Cl}$

Odds ratio

$\begin{array}{llllll}\text { Events } & \text { Total } & \text { Events } & \text { Total } & \mathbf{( \% )} & \text { M-H, fixed, } \\ 12 & 22 & 120 & 160 & 11.8 & 0.40(0.16,1.00)\end{array}$

$\begin{array}{llllll}17 & 20 & 38 & 40 & 3.4 & 0.30(0.05,1.95)\end{array}$

$\begin{array}{ll}40-3.4 & 0.30(0.05,1.95)\end{array}$

$\begin{array}{llllll}44 & 52 & 41 & 54 & 5.5 & 1.74(0.66,4.64)\end{array}$

$\begin{array}{llllll}51 & 72 & 41 & 70 & 10.8 & 1.72(0.86,3.45)\end{array}$

$\begin{array}{llllll}92 & 145 & 87 & 143 & 28.6 & 1.12(0.69,1.80)\end{array}$

$\begin{array}{llllll}47 & 67 & 49 & 66 & 13.2 & 0.82(0.38,1.74)\end{array}$

$30 \quad 33 \quad 33$

$83 \quad 102 \quad 68$

$\begin{array}{lll}41 & 2.4 & 2.42(0.59,9.99)\end{array}$

$103 \quad 11.3 \quad 2.25(1.18,4.28)$

$\begin{array}{llllll}25 & 30 & 23 & 31 & 3.4 & 1.74(0.50,6.09)\end{array}$

$\begin{array}{llllll}99 & 107 & 105 & 110 & 6.9 & 0.59(0.19,1.86)\end{array}$

$\mathrm{Cho}^{20}(2009)$

47

$1.10(0.23,5.26)$

Total $(95 \% \mathrm{Cl})$

701

$853 \quad 100$

$1.21(0.94,1.55)$

Total events $\quad 547 \quad 637$

Heterogeneity: $\chi^{2}=16.76, d f=10(P=0.08) ; I^{2}=40 \%$

Test for overall effect: $Z=1.48(P=0.14)$

$\mathrm{M}-\mathrm{H}$, fixed, $95 \% \mathrm{Cl}$

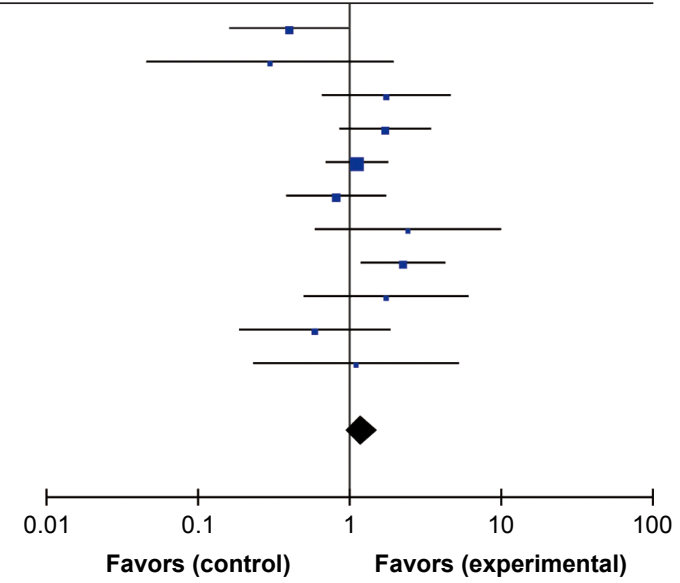

B

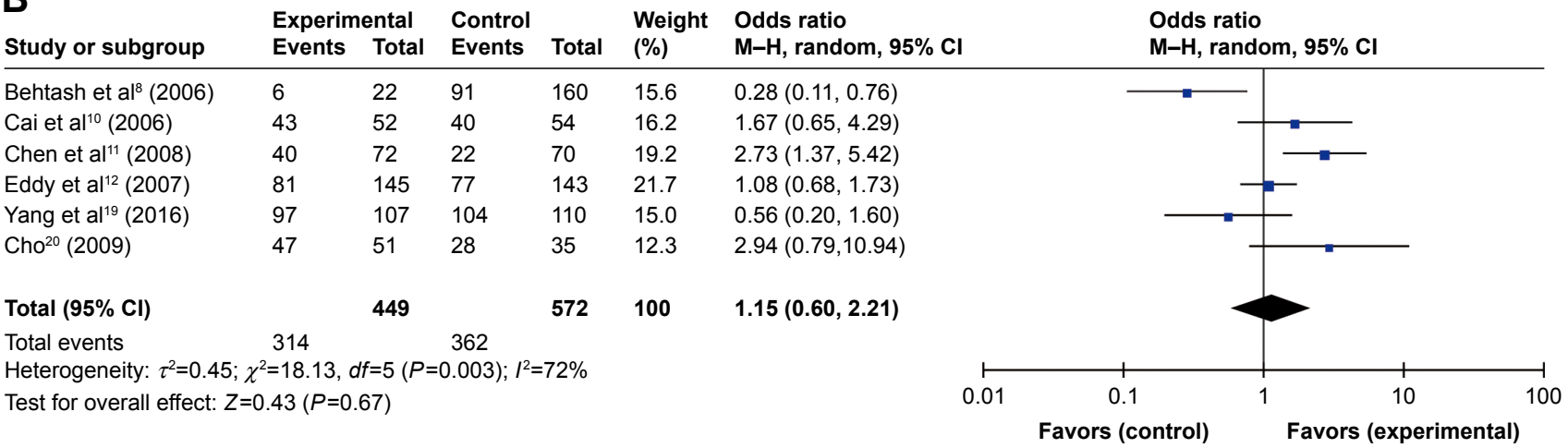

C

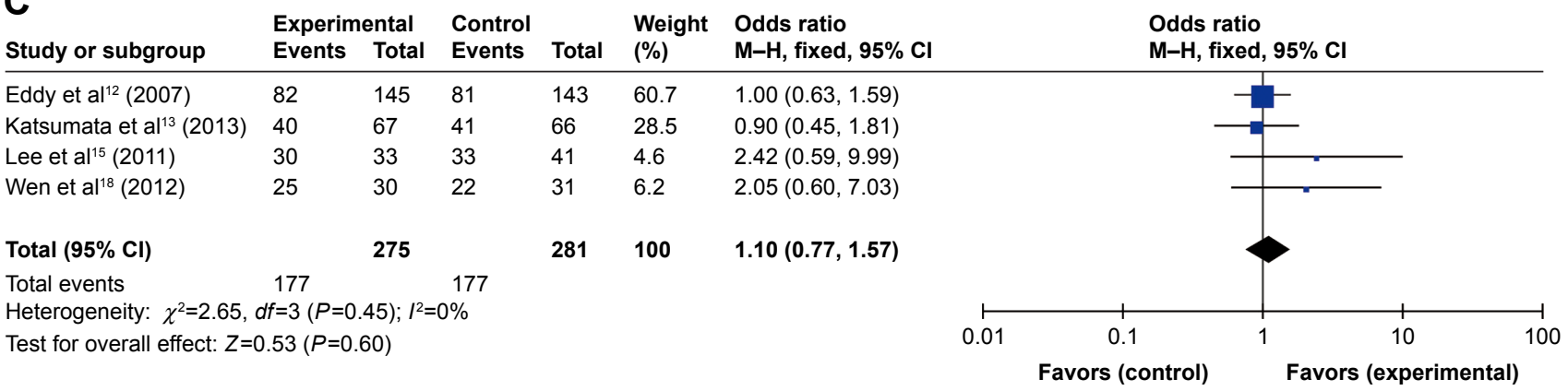

D

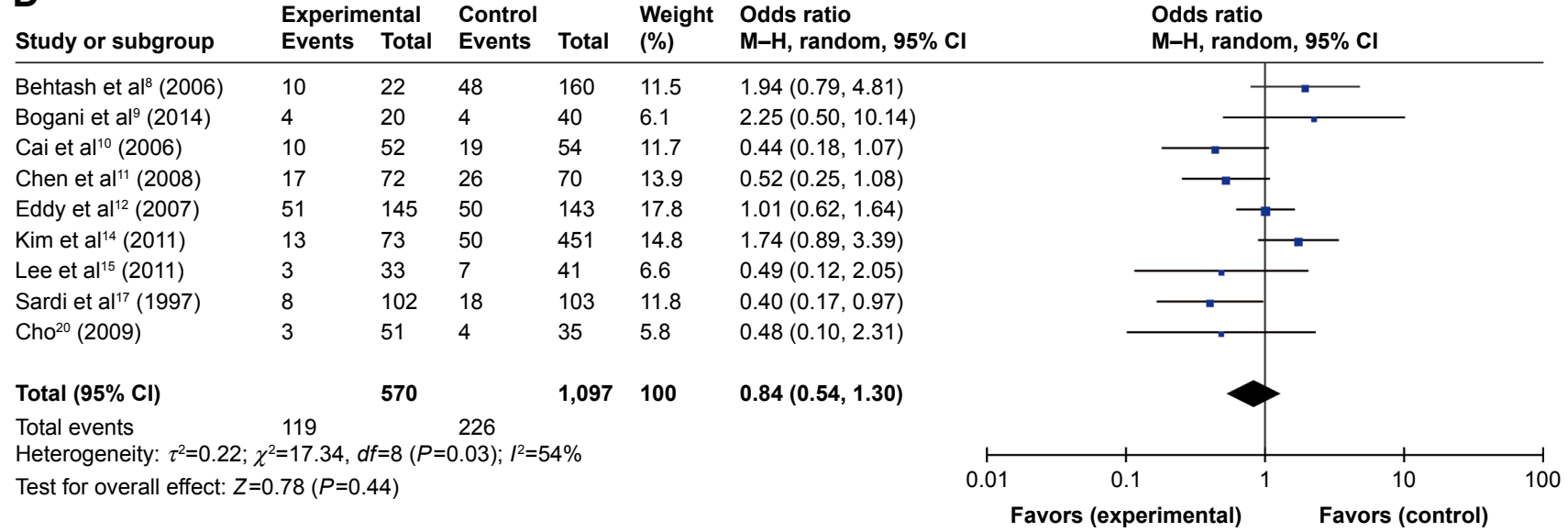

Figure 4 Comparison of long-term efficacy between NACT plus radical surgery group and the radical surgery alone group. (A) Forest plot of overall survival (OS); (B) forest plot of disease-free survival (DFS); (C) forest plot of progression-free survival (DFS); (D) forest plot of recurrence rate.

Abbreviations: M-H, Mantel-Haenszel; NACT, neoadjuvant chemotherapy. 


\section{Local and distant recurrence rates}

Nine studies ${ }^{8-12,14,15,17,20}$ which reported recurrence rate included a total of 1,667 patients (570 in the NACT+radical surgery group and 1,097 in the radical surgery alone group). There was statistical between-study heterogeneity in the OR of studies ( $P=0.03, I^{2}=54 \%$ ); we used the random effects model for merging. The pooled estimates of effect sizes showed no statistical difference in the recurrence rate between the two groups $(\mathrm{OR}=0.84,95 \% \mathrm{CI}=0.54-1.30$, $P=0.44$ ), as shown in Figure 4.

\section{Lymph node metastasis rates}

Twelve studies ${ }^{8-16,18-20}$ reported lymph node metastasis rate, including a total of 1,953 patients (712 in the NACT+radical surgery group and 1,241 in the radical surgery alone group). There was statistical between-study heterogeneity in the OR of studies ( $\left.P=0.01, I^{2}=54 \%\right)$; we used the random effects model for merging. The pooled estimates of effect sizes showed that NACT+radical surgery was associated with a significant decrease in the lymph node metastasis rate compared to radical surgery alone $(\mathrm{OR}=0.63,95 \% \mathrm{CI}=0.44-0.91$, $P=0.01$ ), as shown in Figure 4.

\section{Parametrial infiltration rates}

Ten studies ${ }^{8,10-13,15,16,18-20}$ reported parametrial infiltration rate, including a total of 1,353 patients (613 in the NACT+radical surgery group and 740 in the radical surgery alone group). There was statistical between-study heterogeneity in the OR of studies ( $\left.P=0.009, I^{2}=59 \%\right)$; we used the random effects model for merging. The pooled estimates of effect sizes showed no statistical difference in the parametrial infiltration rate between the two groups $(\mathrm{OR}=0.59,95 \% \mathrm{CI}=0.33-1.05$, $P=0.07)$, as shown in Figure 4.

\section{Subgroup analyses for locally advanced cervical cancer \\ Overall survival}

Seven studies ${ }^{11,13,15,17-20}$ reported OS, including a total of 830 patients (421 in the NACT+radical surgery group, and 409 in the radical surgery alone group). There was no statistical between-study heterogeneity in the OR of studies $\left(P=0.23, I^{2}=27 \%\right)$; we used the fixed effects model for merging. The pooled estimates of effect sizes showed a statistically significant difference in OS between the two groups $(\mathrm{OR}=1.44,95 \% \mathrm{CI}=1.01-2.06, P=0.05)$, as shown in Figure 4.

\section{Local and distant recurrence rates}

Four studies ${ }^{11,13,15,17}$ reported recurrence rate, including a total of 419 patients (217 in the NACT+radical surgery group and 202 in the radical surgery alone group). There was no statistical between-study heterogeneity in the OR of studies ( $\left.P=0.71, I^{2}=0 \%\right)$; we used the fixed effects model for merging. The pooled estimates of effect sizes showed that NACT+radical surgery significantly decreased the recurrence rate compared to radical surgery alone $(\mathrm{OR}=0.43,95 \%$ $\mathrm{CI}=0.25-0.73, P=0.002$ ), as shown in Figure 5.

\section{Lymph node metastasis rates}

Eight studies ${ }^{11,13,15-20}$ reported lymph node metastasis rate, including a total of 910 patients (461 in the NACT+radical surgery group and 449 in the radical surgery alone group). There was no statistical between-study heterogeneity in the OR of studies ( $\left.P=0.15, I^{2}=35 \%\right)$; we used the fixed effects model for merging. The pooled estimates of effect sizes showed that NACT+radical surgery significantly decreased the lymph node metastasis rate compared to radical surgery alone $(\mathrm{OR}=0.46,95 \% \mathrm{CI}=0.34-0.62, P<0.00001)$, as shown in Figure 6.

\section{Parametrial infiltration rates}

Eight studies ${ }^{11,13,15-20}$ reported parametrial infiltration rate, including a total of 910 patients (461 in the NACT+radical surgery group and 449 in the radical surgery alone group). There was statistical between-study heterogeneity in the OR of studies ( $P=0.01, I^{2}=62 \%$ ); we used the random effects model for merging. The pooled estimates of effect sizes showed that NACT+radical surgery significantly reduced for the parametrial infiltration rate compared to radical surgery alone $(\mathrm{OR}=0.54,95 \% \mathrm{CI}=0.29-0.99, P=0.05)$, as shown in Figure 7.

\section{Discussion}

Cervical cancer, one of the most common gynecological malignancies, is a serious threat to women's physical and mental health. Surgery and radiotherapy are recommended for initial therapy of cervical cancer; however, patients still fare poorly in terms of long-term survival. In recent years, cervical cancer has increased in incidence in younger women. In addition, radiotherapy induced vaginal stiffness, narrowing, adhesions, and other complications on the quality-of-life of young patients have gained more and more attention. Previous study has shown that response to NACT is a good surrogate endpoint of survival in patients with locally advanced cervical cancer. ${ }^{21}$ The present study suggests that NACT followed by radical surgery offers a promising alternative to radical surgery alone for patients with locally advanced cervical cancer (FIGO stage IB2-IIB), with a significant improvement in OS, and a marked decrease in the local and 
A

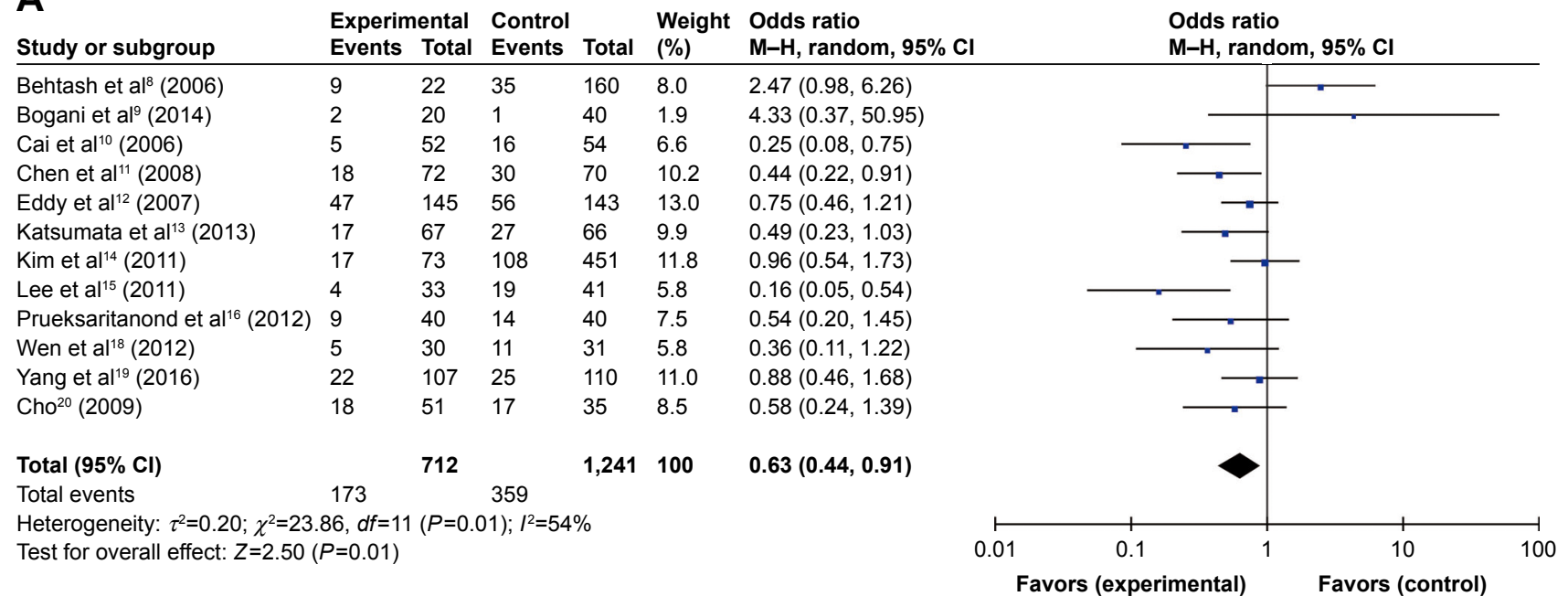

B

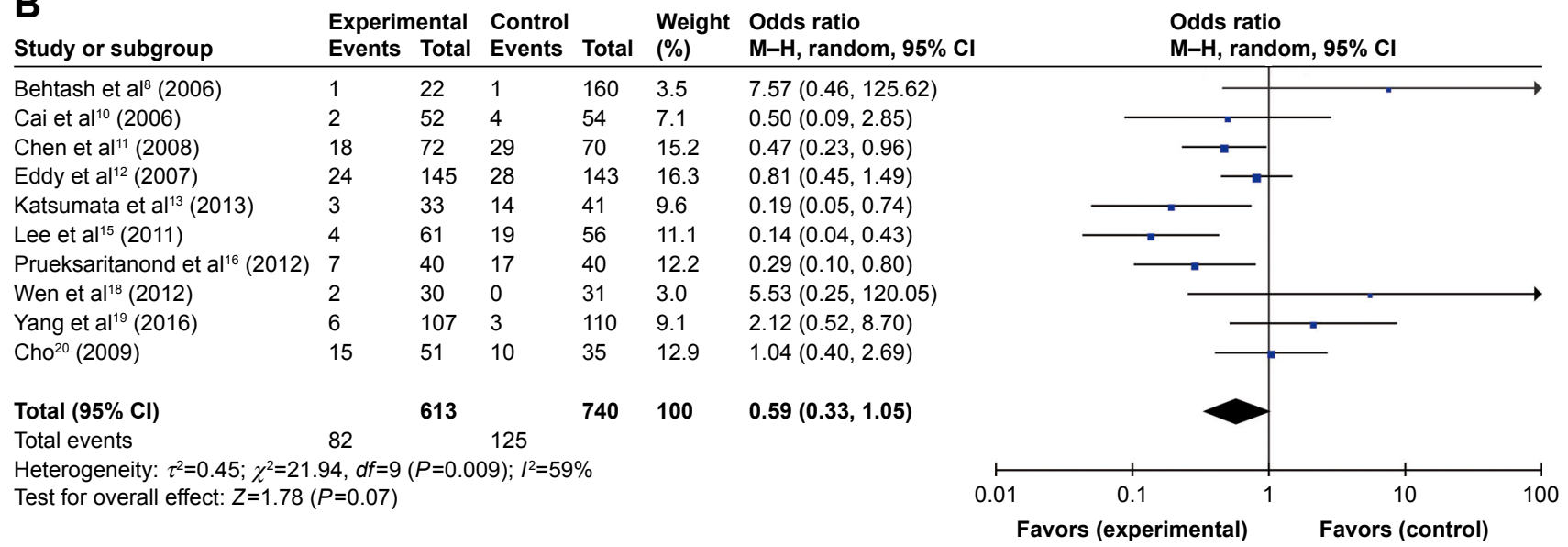

Figure 5 Comparison of pathological findings between the NACT plus radical surgery group and the radical surgery alone group. (A) Forest plot of lymph node metastatic; (B) forest plot of parametrial infiltration.

Abbreviations: $\mathrm{M}-\mathrm{H}$, Mantel-Haenszel; NACT, neoadjuvant chemotherapy.

distant recurrence rate, lymph node metastasis rate, and the level of parametrial infiltration. However, it remains unclear whether NACT plus radical surgery consistently confers a benefit over radical surgery alone on patients with FIGO stage IA and IB1 cervical cancer.

Gadducci et $\mathrm{al}^{22}$ found that the effects of chemotherapy on pathological response $(P<0.0001)$ and FIGO stage $(P=0.0072)$ were statistically significant. There was no statistical significance in age, pathological type (squamous cell carcinoma or adenocarcinoma), tumor diameter $(5 \mathrm{~cm}$ cutoff value), or chemotherapy regimen in a retrospective study of preoperative NACT for locally advanced cervical cancer. Multivariate regression analysis indicated that pathological response was the determinant of long-term prognosis. In this study, Chen et $\mathrm{al}^{23}$ concluded that NACT improved peritumoral infiltration $(P=0.022)$ and lymph node metastasis $(P=0.024)$, but neither NACT nor tumor response to chemotherapy is an independent prognostic predictor. Similarly, Lee et $a^{24}$ compared NACT+surgery with radiotherapy alone/concurrent chemoradiation, and found that, although NACT can reduce the pathological prognosis of locally advanced cervical cancer, and effective response to chemotherapy compared with the ineffective group, the former can improve long-term disease-free survival $(P=0.025)$ and $\mathrm{OS}(P=0.021)$, but also did not improve the long-term prognosis of patients compared to treatment with radiotherapy. Some investigators think that NACT may be employed to improve the prognosis of high risk patients who may fail postoperative consolidation therapy due to occult micro-metastases. It is covered by the "concealing effect hypothesis". ${ }^{25}$ This suggests that an effective chemotherapy response may be used to better predict and improve long-term 
A

\begin{tabular}{|c|c|c|c|}
\hline \multirow{2}{*}{ Study or subgroup } & \multicolumn{2}{|c|}{ Experimental } & \multirow{2}{*}{$\begin{array}{l}\text { Contro } \\
\text { Events }\end{array}$} \\
\hline & Events & Total & \\
\hline Chen et al ${ }^{11}$ (2008) & 51 & 72 & 41 \\
\hline Eddy et al ${ }^{12}(2007)$ & 92 & 145 & 87 \\
\hline Katsumata et $\mathrm{al}^{13}$ (2013) & 47 & 67 & 49 \\
\hline Lee et al ${ }^{15}(2011)$ & 30 & 33 & 33 \\
\hline Sardi et al' (1997) & 50 & 61 & 34 \\
\hline Wen et $\mathrm{al}^{18}(2012)$ & 25 & 30 & 23 \\
\hline Yang et al' ${ }^{19}(2016)$ & 99 & 107 & 105 \\
\hline $\mathrm{Cho}^{20}(2009)$ & 47 & 51 & 32 \\
\hline Total $(95 \% \mathrm{Cl})$ & & 421 & \\
\hline Total events & 349 & & 317 \\
\hline \multicolumn{4}{|c|}{ Heterogeneity: $\chi^{2}=8.18, d f=6(P=0.23) ; I^{2}=27 \%$} \\
\hline
\end{tabular}

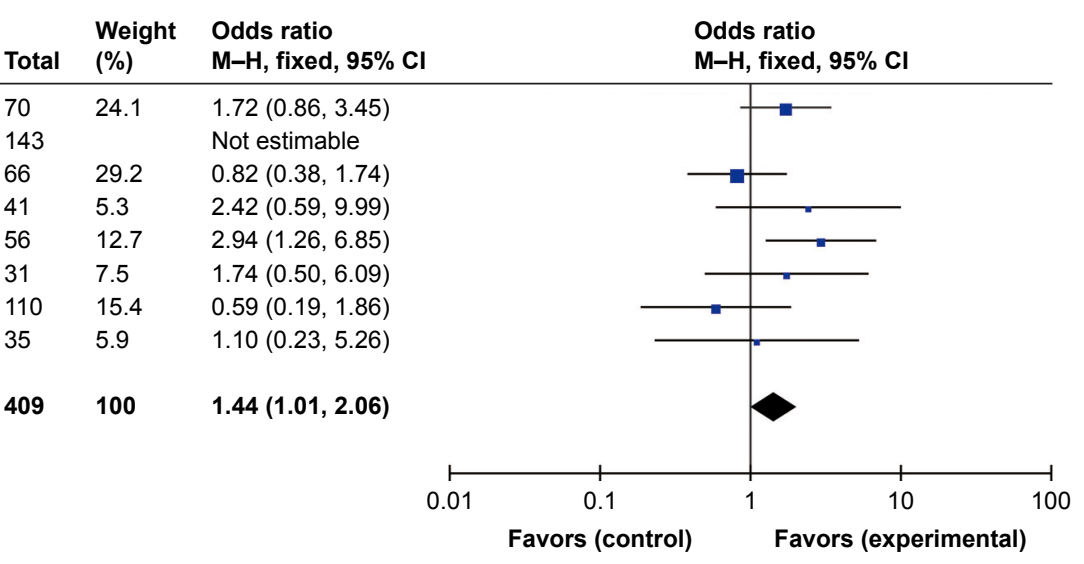

\section{B}

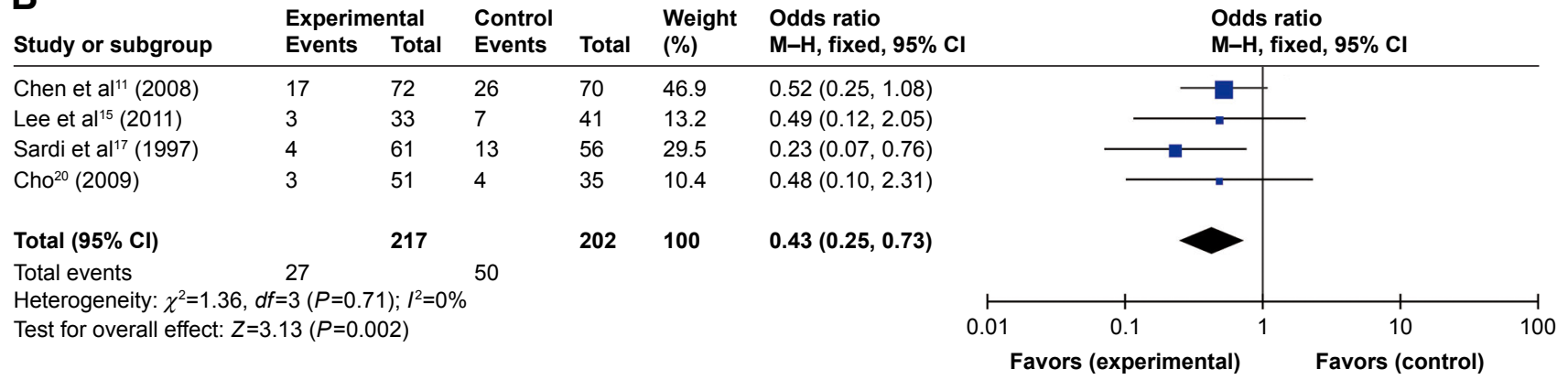

Figure 6 Subgroup analyses of long-term efficacy between the NACT plus radical surgery group and the radical surgery alone group. (A) Forest plot of overall survival (OS); (B) forest plot of recurrence rate.

Abbreviations: $\mathrm{M}-\mathrm{H}$, Mantel-Haenszel; NACT, neoadjuvant chemotherapy.

prognosis of patients with locally advanced cervical cancer who undergo NACT.

According to the postoperative surgical specimens, pathological response was evaluated. The optimal pathological response was $\mathrm{CR}+\mathrm{PR} 1$, which indicates complete disappearance of histological lesions and interstitial infiltration of residual lesions in the neck canal $<3 \mathrm{~mm}$, including carcinoma in situ. However, neither chemotherapy nor radiotherapy significantly improved the long-term prognosis of patients with residual lesions outside the neck canal.

\section{Limitations}

Several limitations of the current analysis should be discussed. First, high response rate is the most important prerequisite for improvement of curative effects. Li et al ${ }^{26}$ suggested that cervical cancer patient response to NACT was an independent determinant of 5-year OS and PFS. Because the original text did not provide enough data, no stratified analysis was done. Second, the 13 researches included in this study are mostly small and/or single-center trials with varying quality. Only two studies had taken the allocation of hidden cases, the allocation of hidden or blind cases was unknown in the remaining studies; six studies with random grouping method were assessed as a high risk of bias, and none of the studies used blinding. In the future, the relevant research needs to be further improved in the following aspects: increase of sample size; right random allocation and allocation of hidden programs; adequate follow-up duration to observe short-term and long-term effects; stratified analysis of chemotherapeutic response rate, tumor staging, volume size as independent factors; and more comprehensive evaluation of the efficacy of NACT.

In summary, the present study demonstrates that preoperative NACT is now an accepted effective procedure in selected patients with locally advanced cervical cancer (FIGO stage IB2-IIB), which is associated with improved OS, and reduced local and distant recurrence rates, lymph node metastasis rate, and the level of parametrial infiltration. However, the relationship between NACT and longer DFS and PFS cannot be demonstrated by this metaanalysis. Thus, the decision to use or not to use NACT before radical surgery depends on the surgeon's experience and clinical judgment. 


\section{A}

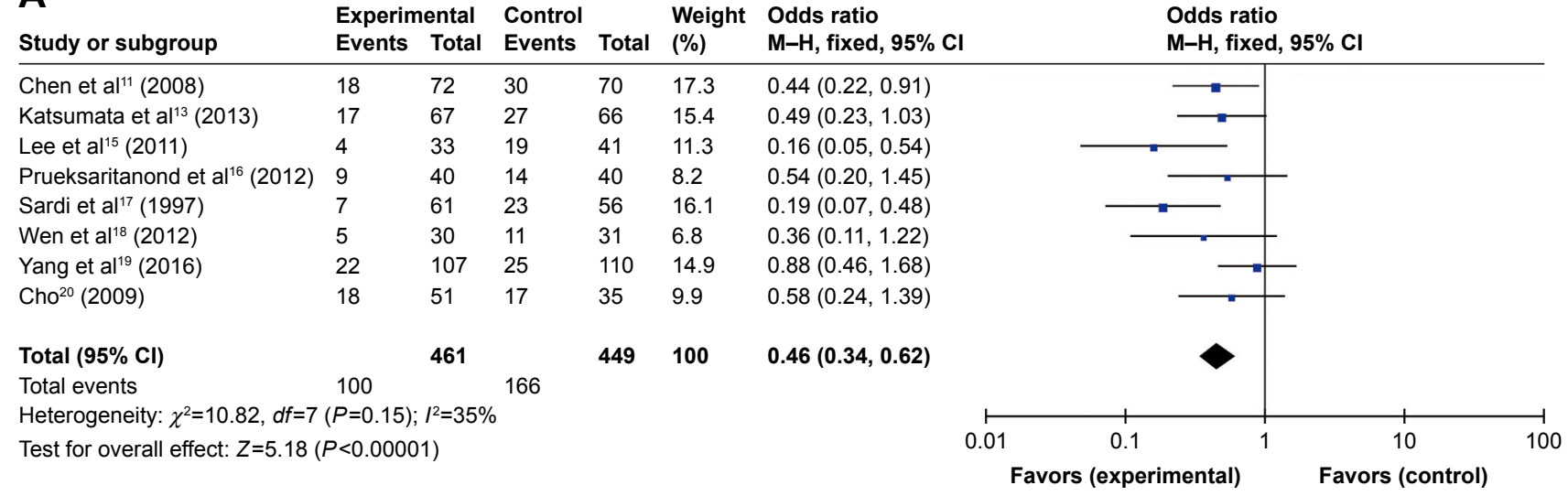

\section{B}

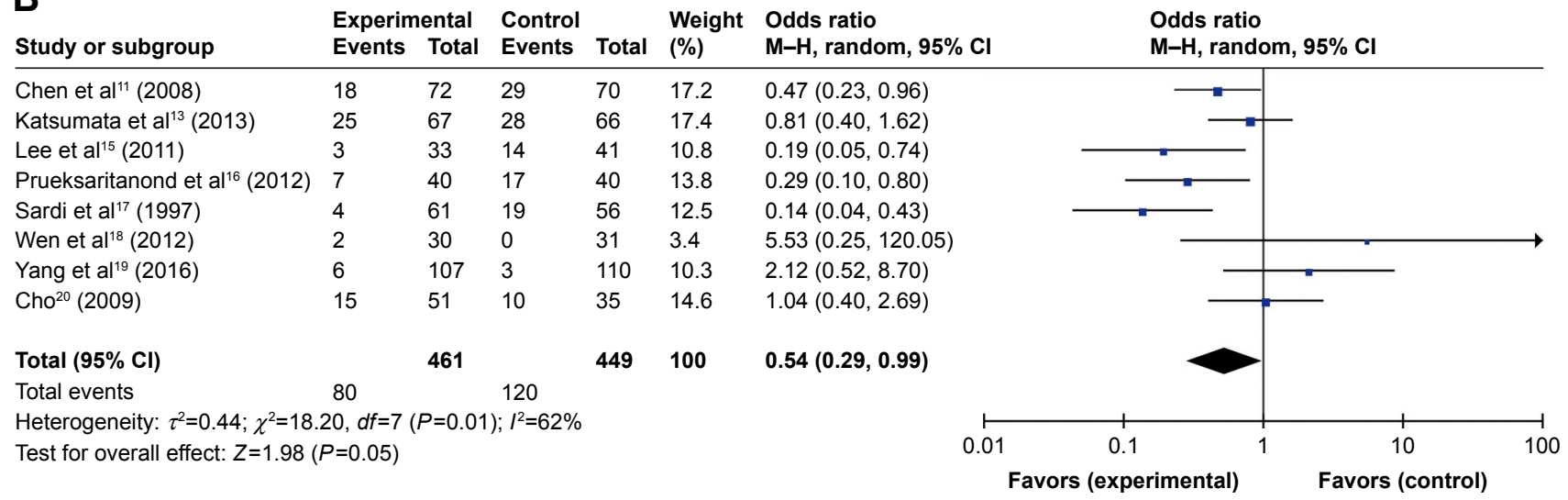

Figure 7 Subgroup analyses of pathological findings between the NACT plus radical surgery group and the radical surgery alone group. (A) Forest plot of lymph node metastatic; (B) forest plot of parametrial infiltration.

Abbreviations: $\mathrm{M}-\mathrm{H}$, Mantel-Haenszel; NACT, neoadjuvant chemotherapy.

\section{Ethical approval}

This article does not contain any studies with human participants performed by any of the authors.

\section{Acknowledgments}

This study was supported by Beijing Municipal Science and Technology Commission (grant nos. D151100001915001 and D131100005313009) and the Special Program for Development of Clinical Medicine of Beijing Municipal Administration of Hospitals (grant no. ZYLX201705).

\section{Disclosure}

The authors report no conflicts of interest in this work.

\section{References}

1. Siegel RL, Miller KD, Jemal A. Cancer statistics, 2016. CA Cancer J Clin. 2016;66(1):7-30.

2. Rose PG. Locally advanced cervical cancer. Curr Opin Oncol. 2001; 13(1):65.

3. Dueñas-González A, Zarbá JJ, Patel F, et al. Phase III, open-label, randomized study comparing concurrent gemcitabine plus cisplatin and radiation followed by adjuvant gemcitabine and cisplatin versus concurrent cisplatin and radiation in patients with stage IIB to IVA carcinoma of the cervix. J Clin Oncol. 2011;29(13):1678-1685.
4. Abe A, Furumoto H, Nishimura M, Irahara M, Ikushima H. Adjuvant chemotherapy following concurrent chemoradiotherapy for uterine cervical cancer with lymphadenopathy. Oncol Lett. 2012;3(3): 571-576.

5. Iii EF. Clinical cancer research: an embattled species. Cancer. 1979; 1982;50(10):1979-1992.

6. Higgins J, Se G, Green SE. Cochrane Handbook for systematic reviews of interventions version 5.1.0. the Cochrane collaboration (EDS). Naunyn-Schmiedebergs Archiv für experimentelle Pathologie und Pharmakologie. 2011;5(2):S38.

7. Liberati A, Altman DG, Tetzlaff J, et al. The PRISMA statement for reporting systematic reviews and meta-analyses of studies that evaluate health care interventions: explanation and elaboration. Epidemiology Biostatistics \& Public Health. 2009;6(4):e1-e34.

8. Behtash N, Nazari Z, Ayatollahi H, Modarres M, Ghaemmaghami F, Mousavi A. Neoadjuvant chemotherapy and radical surgery compared to radical surgery alone in bulky stage IB-IIA cervical cancer. Eur $J$ Surg Oncol. 2006;32(10):1226-1230.

9. Bogani G, Cromi A, Serati M, et al. A prospective case-control study on the impact of neoadjuvant chemotherapy on surgery-related outcomes of laparoscopic radical hysterectomy. Anticancer Res. 2014; 34(10):5703-5708.

10. Cai HB, Chen HZ, Yin HH. Randomized study of preoperative chemotherapy versus primary surgery for stage IB cervical cancer. $J$ Obstet Gynaecol Res. 2006;32(3):315-323.

11. Chen H, Liang C, Zhang L, Huang S, Wu X. Clinical efficacy of modified preoperative neoadjuvant chemotherapy in the treatment of locally advanced (stage IB2 to IIb) cervical cancer: a randomized study. Gynecol Oncol. 2008;110(3):308-315. 
12. Eddy GL, Bundy BN, Creasman WT, et al. Treatment of ("bulky") stage IB cervical cancer with or without neoadjuvant vincristine and cisplatin prior to radical hysterectomy and pelvic/para-aortic lymphadenectomy: a phase III trial of the gynecologic oncology group. Gynecol Oncol. 2007;106(2):362-369.

13. Katsumata N, Yoshikawa H, Kobayashi H, et al. Phase III randomised controlled trial of neoadjuvant chemotherapy plus radical surgery vs radical surgery alone for stages IB2, IIA2, and IIB cervical cancer: a Japan Clinical Oncology Group trial (JCOG 0102). Br J Cancer. 2013 108(10):1957-1963.

14. Kim HS, Kim JH, Chung HH, et al. Significance of numbers of metastatic and removed lymph nodes in FIGO stage IB1 to IIA cervical cancer: primary surgical treatment versus neoadjuvant chemotherapy before surgery. Gynecol Oncol. 2011;121(3):551-557.

15. Lee JY, Kim YH, Kim MJ, et al. Treatment of stage IB2, IIA bulky cervical cancer: a single-institution experience of neoadjuvant chemotherapy followed by radical hysterectomy and primary radical hysterectomy. Arch Gynecol Obstet. 2011;284(2):477-482.

16. Prueksaritanond N, Chaisarn P, Yanaranop M. The efficacy of neoadjuvant paclitaxel-carboplatin chemotherapy followed by radical hysterectomy compared to radical hysterectomy alone in bulky stage IB2-IIA cervical cancer. J Med Assoc Thai. 2012;95(Suppl 3):S55.

17. Sardi JE, Giaroli A, Sananes C, et al. Long-term follow-up of the first randomized trial using neoadjuvant chemotherapy in stage IB squamous carcinoma of the cervix: the final results. Gynecol Oncol. 1997; 67(1):61-69.

18. Wen $\mathrm{H}, \mathrm{Wu} \mathrm{X}, \mathrm{Li} \mathrm{Z}$, et al. A prospective randomized controlled study on multiple neoadjuvant treatments for patients with stage IB2 to IIA cervical cancer. Int J Gynecol Cancer. 2012;22(2):296-302.
19. Yang Z, Chen D, Zhang J, et al. The efficacy and safety of neoadjuvant chemotherapy in the treatment of locally advanced cervical cancer: a randomized multicenter study. Gynecol Oncol. 2016;141(2):231-239.

20. Cho YH, Kim DY, Kim JH, Kim YM, Kim YT, Nam JH. Comparative study of neoadjuvant chemotherapy before radical hysterectomy and radical surgery alone in stage IB2-IIA bulky cervical cancer. J Gynecol Oncol. 2009;20(1):22-27.

21. van Dam PA, Rolfo C, Ruiz R. Neoadjuvant trials can accelerate research on novel systemic treatment modalities in cancer of the uterine cervix. Eur J Surg Oncol. 2017;43(12):2245-2247.

22. Gadducci A, Sartori E, Maggino T, et al. Pathological response on surgical samples is an independent prognostic variable for patients with stage Ib2-IIb cervical cancer treated with neoadjuvant chemotherapy and radical hysterectomy: an Italian multicenter retrospective study (CTF study). Gynecol Oncol. 2013;131(3):640-644.

23. Chen CA, Cheng WF, Wei LH, Su YN, Hsieh CY. Radical hysterectomy alone or combined with neoadjuvant chemotherapy in the treatment of early stage bulky cervical carcinoma. J Formos Med Assoc. 2002 ; 101(3):195-202.

24. Lee DW, Lee KH, Lee JW, Park ST, Park JS, Lee HN. Is neoadjuvant chemotherapy followed by radical surgery more effective than radiation therapy for stage IIB cervical cancer? Int J Gynecol Cancer. 2013;23(7):1303-1310.

25. Kim K, Kim MJ, Chung HH, et al. Inadvertent potential risk of neoadjuvant chemotherapy in cervical cancer. Med Hypotheses. 2009; 73(6):1005-1007.

26. Li R, Lu ST, Si JG, et al. Prognostic value of responsiveness of neoadjuvant chemotherapy before surgery for patients with stage IB(2)/IIA(2) cervical cancer. Gynecol Oncol. 2013;128(3):524-529.
OncoTargets and Therapy

\section{Publish your work in this journal}

OncoTargets and Therapy is an international, peer-reviewed, open access journal focusing on the pathological basis of all cancers, potential targets for therapy and treatment protocols employed to improve the management of cancer patients. The journal also focuses on the impact of management programs and new therapeutic agents and protocols on

\section{Dovepress}

patient perspectives such as quality of life, adherence and satisfaction. The manuscript management system is completely online and includes a very quick and fair peer-review system, which is all easy to use. Visit http://www.dovepress.com/testimonials.php to read real quotes from published authors. 